\title{
A Future of Current Flow Modelling for Transcranial Electrical Stimulation?
}

\author{
J. S. A. Lee ${ }^{1} \cdot$ S. Bestmann ${ }^{1,2} \cdot$ C. Evans ${ }^{1}$
}

Accepted: 25 September 2021

(c) The Author(s) 2021

\begin{abstract}
Purpose of Review Transcranialelectrical stimulation (tES) is used to non-invasively modulate brain activityin health and disease. Current flow modeling (CFM) provides estimates of whereand how much electrical current is delivered to in the brain during tES. Ittherefore holds promise as a method to reduce commonplace variability in tESdelivery and, in turn, the outcomes of stimulation. However, the adoption ofCFM has not yet been widespread and its impact on tES outcome variability isunclear. Here, we discuss the potential barriers to effective, practicalCFM-informed tES use.

Recent Findings CFMhas progressed from models based on concentric spheres to gyri-precise headmodels derived from individual MRI scans. Users can now estimate the intensityof electrical fields (E-fields), their spatial extent, and the direction ofcurrent flow in a target brain region during tES. Here. we consider the multi-dimensionalchallenge of implementing CFM to optimise stimulation dose: this requiresinformed decisions to prioritise E-field characteristics most likely to resultin desired stimulation outcomes, though the physiological consequences of themodelled current flow are often unknown. Second, we address the issue of adisconnect between predictions of E-field characteristics provided by CFMs andpredictions of the physiological consequences of stimulation which CFMs are notdesigned to address. Third, we discuss how ongoing development of CFM inconjunction with other modelling approaches could overcome these challengeswhile maintaining accessibility for widespread use.

Summary Theincreasing complexity and sophistication of CFM is a mandatory step towards dosecontrol and precise, individualised delivery of tES. However, it also riskscounteracting the appeal of tES as a straightforward, cost-effective tool forneuromodulation, particularly in clinical settings.
\end{abstract}

Keywords Transcranial electrical stimulation (tES) · Current flow modelling (CFM) · Neurophysiology · Therapeutic tES · Non-invasive brain stimulation (NIBS)

Modulation of brain activity via surface electrodes attached to the scalp holds great appeal as a non-invasive, inexpensive, safe, and, prima facie, easy to use method of brain stimulation [1-3]. Unsurprisingly, transcranial electrical stimulation (tES) has attracted enormous interest over the past two decades and holds potential for therapeutic use across a wide range of neurological and psychiatric disorders, including but not limited to Parkinson's disease [4], stroke [5-7], depression [8-10], addiction [11, 12], and Alzheime's disease [13]. However, tES outcomes are dogged

C. Evans

carys.evans@ucl.ac.uk

1 Department for Clinical and Movement Neurosciences, UCL Queen Square Institute of Neurology, 33 Queen Square, London WC1N 3BG, UK

2 Wellcome Centre for Human Neuroimaging, UCL Queen Square Institute of Neurology, London, UK by inter- and intra-individual variability, often small effect sizes, and limited efficacy [14-17], which impede its widespread and effective adoption into clinical practice.

The advent of current flow modelling (CFM), and increased efforts open-access, user-friendly application, boost the reliability, reproducibility, and efficacy of tES. CFM provides estimates of the distribution and intensity of electric fields (E-fields) in the brain during stimulation, allowing users to design model-based tES protocols to target specific brain areas or networks. Consequently, CFM has been heralded as a way for controlling tES delivery both within and across subjects and as a means to curb the variable outcomes that currently bedevil the field.

Yet, decades since the introduction of MRI-derived models [18-20], has CFM improved tES outcomes? The short answer to this question is likely that it has not; CFM has not yet been 
adopted into wider practice and has not become part of the routine for targeted tES delivery, nor for informing rationales for research and clinical application of tES.

We here discuss three determinant issues for the future of CFM in tES research and clinical use. First, we consider the multi-dimensional problem of optimisation of tES delivery. Second, we discuss how likely it is that CFMs will help us to predict target engagement and behavioural stimulation effects, given that CFMs are not designed for predicting the physiological impact of tES. Third, the technological demands and mandatory expertise required for the application of CFM (in combination with other models and approaches) are rapidly growing. We ask if the advancing sophistication of CFMs, potentially in conjunction with models that can predict physiological consequences of tES with high precision, will render the technique inaccessible for widespread clinical use?

\section{Functionality of Current Flow Models}

Current flow models simulate tES application and provide estimates of the magnitude, distribution, and direction of current that a given protocol will deliver to the brain. This is achieved through classification of different tissue types, based on MRI scans [21], and the assignment of conductivity values to the segmented tissue compartments (e.g. skin, scalp, CSF, grey matter, white matter). This allows for predicting the properties of current flow throughout the head [22, 23]. Previously modelled on concentric spheres [20, 24, 25] or standard model heads [26], CFMs today use individual structural MRI to inform researchers of where and how much current is likely to reach different parts of the brain, given a specific electrode montage and stimulation intensity applied to an individual [27-34].

CFMs have now been validated in a range of studies, including comparison with in vivo human and non-human primate recordings during application of tES [18, 35-38]. While nuanced differences distinguish between different CFM approaches, they generally provide, gyri-precise estimates of E-fields in the brain.

We here focus on two attributes of CFM. First, CFMs can provide estimates of where and how much current is delivered to in the brain given a user-defined tES protocolinformation that cannot be obtained non-invasively by other means. Alternatively, CFMs can guide tES protocol selection given a set of criteria, such as desired magnitude or direction of E-field in a cortical region of interest (Fig. 1).

\section{What Are the Optimisation Criteria for Effective tES Delivery?}

The inter- and intra-individual effects of tES remain variable $[14,16,17,39,40]$, as do the protocols used between different studies. CFMs can be used to minimise inter-individual variability in the magnitude, focality, or direction of current at a cortical target, though they are agnostic to the effects associated with current applied to the brain. By exploiting Ohm's law, for example, one can use the predictions of CFM to adjust stimulator output for each subject such that dose in a cortical target area is matched across all individuals [41]. However, the CFM would not provide predictions of the physiological outcome of the simulated protocol.

The importance of critical decision-making by the CFM user is apparent; tES optimisation might only be achieved where a user is able to rationally prescribe tES parameters

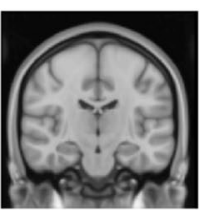

1. MRI

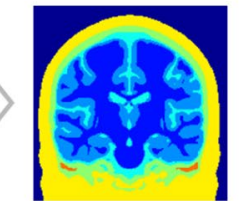

2. Segment
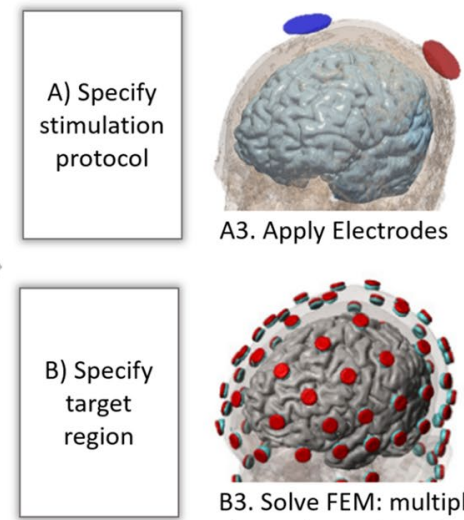

A3. Apply Electrodes

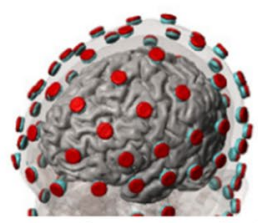

B3. Solve FEM: multiple electrode combinations

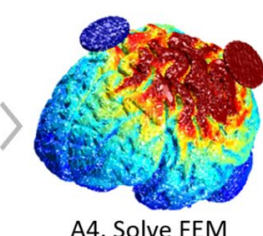

A4. Solve FEM

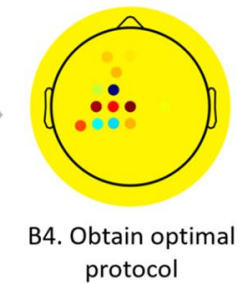

Fig. 1 Current flow modelling. An individual MRI (1) is segmented into different tissue types (2). If a stimulation protocol is specified (A3), the finite element model (FEM) for this specific electrode montage is solved (A4). If target E-field properties are specified, the FEM is solved for multiple electrode combinations (B3), to identify a stimulation protocol that optimises tES application to achieve the desired criterion (for example a montage that delivers focal stimulation to a cortical target region) (B4). Image B3 modified from Huang et al. 2019 [31] 
likely to achieve a desired effect. This may be possible when sufficient precedence exists in the literature for the expected effects of a given tES protocol. However, it is though often unclear which E-field characteristics should be prioritised to target a given brain region (is the direction or intensity of current in the brain more predictive of tES outcome?), or what the optimal parameters for CFM application might be (what intensity of stimulation should be applied to the scalp?). While CFMs are useful for post hoc assessment of brain regions which have likely been exposed to currents above a given threshold [42], for novel questions and applications, the parameters likely to yield the strongest and most reliable effects are often unknown.

Let us assume, for example, that a given tES montage applied at $1 \mathrm{~mA}$ was to create, on average, an E-field magnitude of $0.2 \mathrm{~V} / \mathrm{m}$ (and consequently $2 \mathrm{~mA}$ would deliver, on average, $0.4 \mathrm{~V} / \mathrm{m}$ ) to a primary region of interest (ROI) in the brain. Across individuals, the E-field magnitude in the ROI would likely vary substantially, by over $100 \%$ or more $[18,35,38,41,43]$. Assuming that the E-field magnitude in the ROI is the determining factor for the effect on neural processing, tES optimisation could be achieved by a simple adjustment of the stimulator intensity in each person, based on their individual CFM, such that the dose (e.g. $0.2 \mathrm{~V} / \mathrm{m}$ ) is the same for all individuals. This would remove the inter-individual variance originating from differences in the applied dose. It appeals to intuition that doing so should also remove (some of) the variance in the effects of stimulation. However, whether this is indeed the case and, consistently so, remains to be shown.

Similarly, one may be tempted to increase stimulator output with the goal of maximising E-field magnitude in an ROI (while staying within safety limits). Conceptually, there is little merit in this approach because the dose-response relationship of human in vivo tES is likely non-monotonic [44, 45], and the optimal E-field magnitude for target engagement is generally not known. Simply maximising current for everyone, up to an acceptable stimulator output intensity, is unlikely to result in predictable or reliable effects. Drawing rationales from animal studies may have limited value because the intensities used in animal models are often much higher $[44,46]$ and current delivery more tightly controlled [47] than is possible in human applications. While technically, therefore, CFMs can now be used to adjust stimulator output to specific values for each individual, it remains unclear how one should adjust stimulation protocols. Simply assuming that larger stimulation intensities will lead to larger effect sizes is likely to be incorrect.

To add complexity, the E-field magnitude is not the only variable that determines the impact of stimulation on physiology. Increasing stimulator output will inevitably also increase the number of brain regions exposed to E-field magnitudes equal or above values expected in a targeted region.
It would seem judicious to assume that inter-individual variability in both the magnitude and spatial distribution of current contributes to variability in the physiological outcomes of tES. Controlling for both variables at once poses a more significant optimisation problem for CFM than optimising for the magnitude of the electrical field alone.

The vexed issue of spatial distribution of current is grounded in the idea that focal stimulation is generally desirable, but that tES, by delivering diffuse current, is inevitably not focal. We point out that it is by no means established that focal stimulation is indeed necessary, or even important, for efficacious stimulation, but for simplicity, we will here assume that focal delivery can at least simplify optimisation of tES delivery.

Two approaches can be used to limit the spatial extent of E-field throughout the brain. First, one can simply reduce stimulator output intensity. This results in exposure of fewer brain regions to current above a certain threshold. Second, the use of multi-electrode tES montages can constrain diffuse current $[18,28,48,49]$. An example of this approach is the so-called $4 \times 1$ montage where an electrode (e.g. an anode) placed on the scalp over a cortical region of interest is encircled by a ring of electrodes (e.g. cathodes). Such a montage constrains the spread of current radiating from the central electrode [18, 24]. This exemplifies the contribution of CFMs which have provided suggestions for montages that minimise spatial spread of E-fields, both within and between subjects.

However, the increased focality that can be achieved with multi-electrode montages must be balanced against reduced E-field magnitude (given the same stimulator output) resulting from increased shunting between proximal electrodes on the scalp [19,28], and increased E-field variability [50] (Fig. 2). In principle, this trade-off can be mitigated by adjusting the stimulator output, though matching E-field magnitudes achieved by bipolar tES may require stimulation intensities that exceed what is commonly tolerated or within recommended safety limits.

Finally, the direction of current relative to the orientation of targeted neural structures is a critical though seldomdiscussed determinant of tES effects. In single neurons, the direction of applied current with respect to the somato-dendritic axis of a target neuron dictates polarisation effects [46, 51-53]: as an approximation, current flowing radial-inward from dendrite to soma causes somatic depolarisation and is associated with excitation of the cell, whereas current flowing radial-outward from soma to dendrite causes hyperpolarisation and is associated with inhibition. Tangential current flow does not induce somatic polarisation (Fig. 3).

CFM suggests that conventional tES application, where electrodes are positioned on the scalp directly over a cortical target site, likely produces varied and predominantly tangential current in targets positioned in gyral banks of the brain (such as the primary motor cortex (M1), a common target for tES) [52, 53]. CFMs offer some remedy to the issue as 
Fig. 2 E-field when modelling bipolar and HD $(4 \times 1)$ electrode montages. (A) higher intensities achieved with bipolar montage at the cost of focality. (B) improved focality at the cost of intensity. C) E-field direction plotted around the central sulcus (image $\mathbf{C}$ taken from Saturnino et al., 2019) [34]
A) INTENSITY

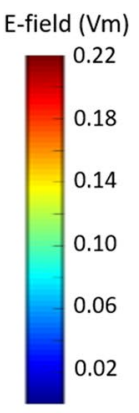

B) FOCALITY

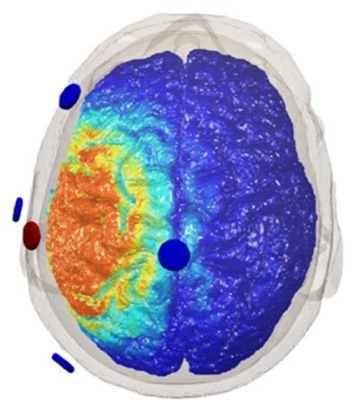

C) DIRECTION

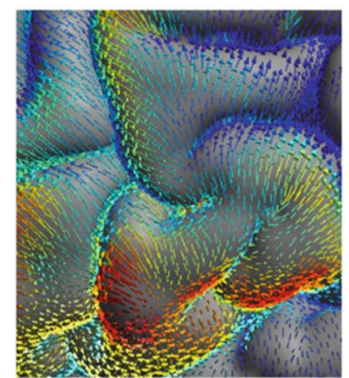

Fig. 3 Polarisation effect of applied current depending on neuron orientation. Current applied radial-inward along the somato-dendritic axis from dendrite to soma causes depolarisation (D), current applied radial-outward from soma to dendrite causes hyperpolarisation $(\mathrm{H})$, and current applied tangentially across the somatodendritic axis causes little to no polarisation effect

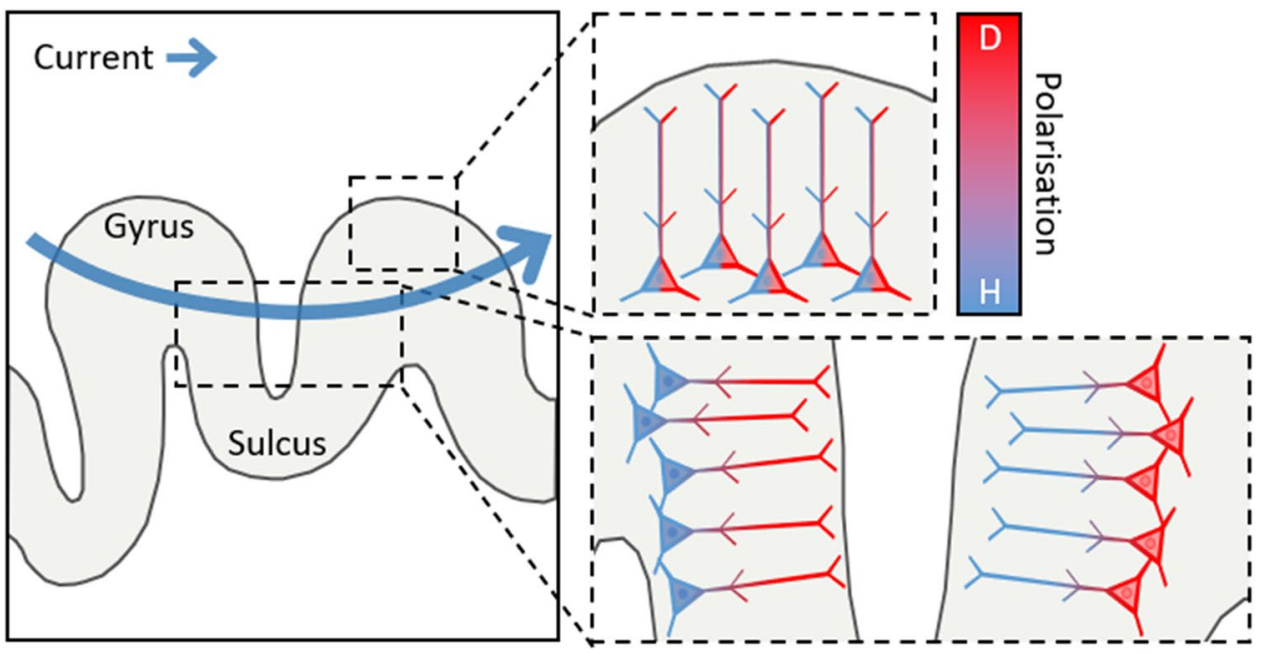

they can be used to estimate the direction of current in relation to the somato-dendritic axes of pyramidal neurons, for example, by using the cortical surface at a given location as proxy for the predominant orientation of pyramidal neurons at a target site. Application of tES can then be optimised to achieve more consistent E-field direction in an ROI across a group of individuals. This can indeed lead to greater consistency in tES effects $[54,55]$. Such optimisation can be done in combination with adjustment of the stimulation intensity, though simultaneously controlling for a third parameter, the spatial distribution of current, is a much harder optimisation problem (Fig. 2).

Nevertheless, the strength of CFMs lies in their ability to generate novel predictions about where and how much current is delivered to in the brain with a given montage, and to thereby control the dosing of tES intervention. This, in turn, has spawned further development and more recent incarnations of CFM software can, for example, deal with multiple ROIs at once [32]. This is relevant as the diffuse nature of tES means many brain regions are inevitably exposed to current. Without CFMs, one would be reduced to guessing which regions are targeted by a given tES protocol.

Going forward, CFM has the potential to drastically change the way we administer tES (it has cautiously done so already). It can facilitate a move away from conventional fixed stimulation protocols based on historical norms, towards informed, individualised tES delivery. In practice, the optimisation problem is multi-variate in nature, with inter-dependencies between magnitude, spatial distribution, and direction of current delivered to the brain. Some constraints are inescapable, such as limits to the intensity of current that can be delivered, or the focality (or lack thereof) that can be accomplished with tES, but these can provide useful constraints to optimisation. While CFM can therefore be used to control the magnitude, focality, or direction of current at a cortical target, currently, there is no straightforward optimisation routine available to optimise for all three variables at once. Informed tES study design thus remains a balancing act, which relies as heavily on judgement calls by the researcher as it does on the information provided by CFMs.

The critical issue, however, is another: even if CFM users could optimise across the parameter space, we would remain naïve to the physiological consequences of the modelled tES protocol and the degree of target engagement. The question then is whether - even if we knew how to optimise tES delivery - the variability of outcomes could be reduced in a meaningful way? Will 
the use of models that are agnostic to physiology ever be sufficient to develop effective and reliable rationales for stimulation protocols?

\section{How Generalisable Are CFMs?}

The utility of CFMs lies in generating predictions about the properties of tES currents flowing through the brain. However, there is currently no straightforward mapping between the diffuse currents delivered by tES, their physiological impact on the brain, and the resulting behavioural consequences of stimulation. Here, we will focus on two factors which require attention because they determine what stimulation effects will be but are rarely taken into account when designing stimulation protocols: the anatomical and physiological state or trait of the subject.

CFMs provide estimates of current flow based on assumptions about the 'anatomical state' of healthy individuals, for example, anatomical features and tissue properties of the healthy adult brain. That anatomical state can vary profoundly across different populations is not considered. Put simply, standard CFMs assign the same tissue conductivity values to all populations and are agnostic to changes which occur across the lifespan or in pathology. For example, the impact of morphological changes associated with healthy ageing $[39,56-59]$ on the distribution of current is not known.

Structural changes that occur in degenerative disease such as dementia or ALS, or in acquired brain injury such as stroke, are also likely to have an impact on the distribution of current such that optimisation criteria for tES derived from healthy populations may no longer apply [60, 61]. We know that changes in skull density, and in thickness of CSF and skull tissue [62, 63], or the presence of lesions [61, 64] will drastically alter E-field distribution. For example, the presence of a lesion can result in either an increase or decrease in E-field magnitude in a region of interest, depending on the lesion location and size relative to the path of the current [60]. To add to this complexity, the conductances of lesioned tissue also remain unknown [60; 81]. The likely impact of these brain differences on dose and target engagement poses a challenge to the efficacy of stimulation in clinical practice $[62,65]$.

The extent to which predictions of CFMs generalise to other populations remains untested, perhaps simply because accommodating for structural differences across different clinical populations is not straightforward and currently requires bespoke solutions with considerable modelling expertise. Even if CFMs accounted for anatomical state, however, they would not offer predictions of the physiological impact of target engagement.
A similar argument can be made for the 'functional state' of the brain, which we here simplistically equate with the state of the underlying activity, neurochemistry, or excitability in a targeted region or network at the time of stimulation. Consider the simple case of applying tES either during task performance or during rest. Clearly, brain activity in these two states differs, and tES application will result in a different response: applying tES either before or during movement can result in opposing effects [66-69], though CFMs would prescribe an identical stimulation protocol in either case.

Another example pertains to the influence of neurotransmitters on the impact of stimulation, and vice versa. On the one hand, we know that tES can alter GABA concentration, even in unstimulated brain regions [70, 71], and that this directly relates to performance in a behavioural task [72-74]. We also know that neurochemical 'state' determines the capacity to undergo tES-induced cortical plasticity $[45,75,76]$. For example, the relative concentrations of both GABA and glutamate in prefrontal cortex determine the impact of tES on performance in a response to a selection task [77]. Since CFMs are not designed to account for functional state and trait differences, they cannot be used in isolation to support a priori optimisation, especially in cases where the underlying state of the brain is likely to influence target engagement.

Target engagement to any tES intervention depends on functional state during stimulation. Yet we (almost exclusively) select our clinical stimulation protocols from studies conducted in healthy young adults. For example, many of the neurological or psychiatric disorders studied and treated with $\mathrm{tES}$ are characterised by abnormalities in one or several neurotransmitter systems. The expectation that the effects of stimulation in patients can be predicted from the effects observed in healthy individuals receiving the same intervention seems fraught with logical problems (see Fig. 4 for hypothetical dose-response relationships in health and pathology).

Indeed, even if clinical anatomical differences were successfully accounted for through CFMs, for example, by development of models which incorporate lesions and their effect on current flow, the missing link to target engagement will ultimately necessitate the addition of adjunct models and measurements. Biophysically and morphologically realistic models are now emerging [78], and biophysically grounded computational models that are able to predict some of physiological and behavioural tES outcomes are being developed $[79,80]$. These can formulate mechanistically grounded predictions about the impact of stimulation. Similarly, neuroimaging is one way to demonstrate target engagement, though in isolation it will not inform about the efficacy of the intervention on behaviour.

CFMs have facilitated a recent critical jump in understanding of tES application, though when used in isolation, this may not be sufficient to increase reliability and 
A)

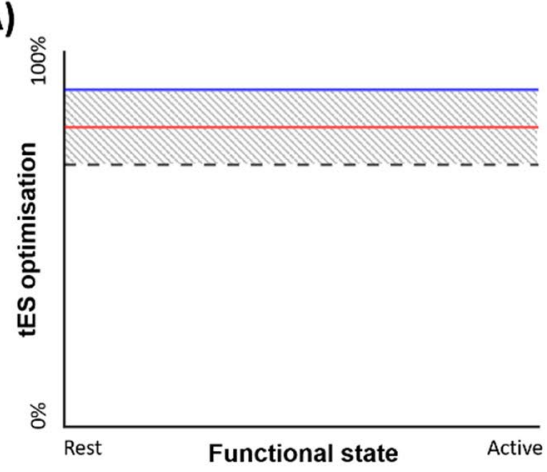

D)

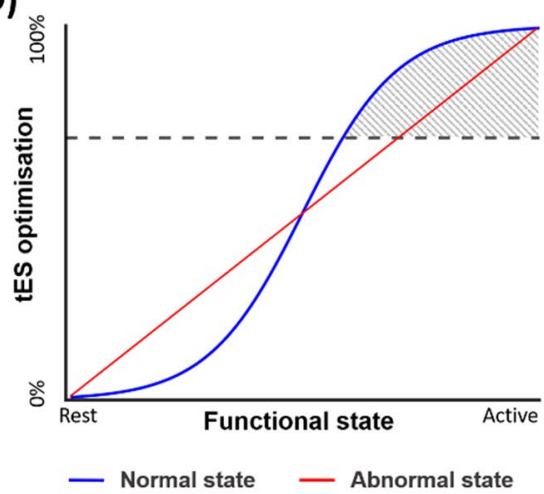

B)

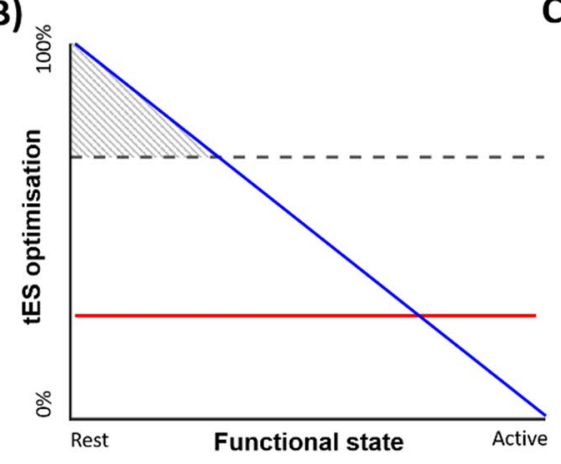

E)

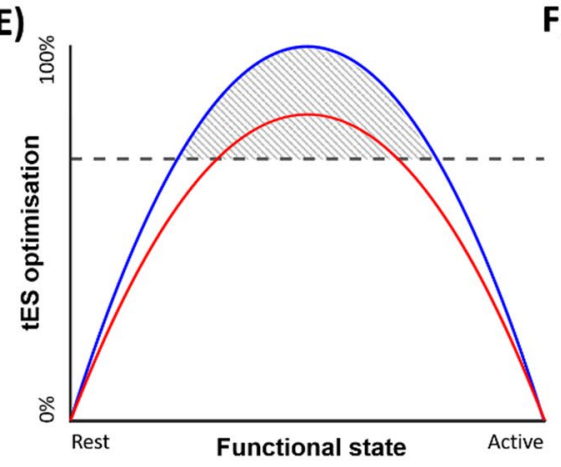

C)

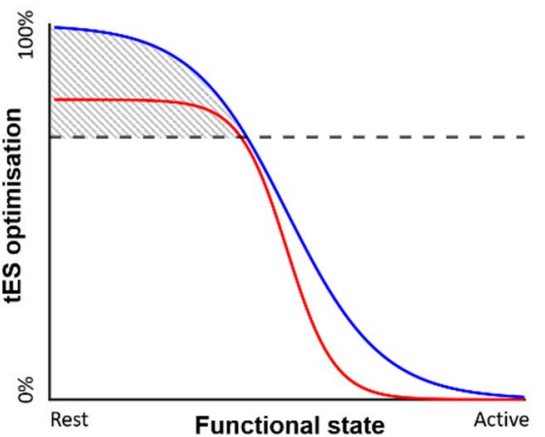

F)

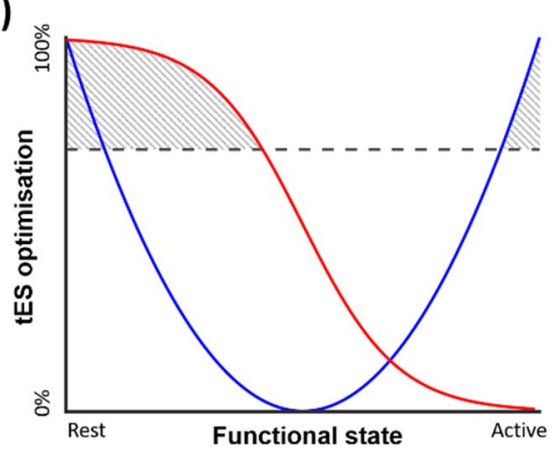

Fig. 4 Possible dose-response relationships between E-field and functional state of the brain. The effect of stimulation is determined by the magnitude, focality, and direction of E-field (tES optimisation: 0 to $100 \%$ ), in interaction with the functional state of the brain (functional state: rest to active). The dashed line denotes a threshold for human neuromodulation (value unknown), above which a meaningful stimulation effect is expected (shaded grey area). In general, the function that maps the stimulation parameters onto tES outcomes in either health (blue lines) or disease (red lines) is unknown, with some but not all possible cases depicted in the figure. (A) The common assumption is that once a threshold for neuromodulation is reached, the effects are constant regardless of state. (B-D). The effects of stimulation are state-dependent, being largest either at rest $(\mathbf{B}, \mathbf{C})$ or in the active state (D). (E-F) The mapping between stimulation and its effects is non-monotonic or non-linear

and direct current in a desired orientation relative to a target structure. These parameters can be prescribed by the researcher or suggested by the model itself.

CFMs are thereby facilitating a move away from historical norms which prevent mechanistically informed study design, such as binary ideas of stimulation effects propagated by the language 'anodal' and 'cathodal' tES. They have enabled a shift from group-level design-applying a fixed intensity and electrode montage to everyonesonalised tES application. Within-group variability, often masked by group-level analyses, can now be quantified and reduced.

That said, it is by no means clear that CFMs improve stimulation outcomes. Research showing a relationship between E-field magnitude in a cortical target and physiological outcomes hints that we are on the right track [42]. Yet it remains unclear which parameters should be prioritised by the CFM user for optimisation, and how these parameters differ across different populations with fundamental anatomical and functional differences. A greater number 
of CFM-informed tES studies, which control for the multivariate characteristics of endogenous current (magnitude, spatial distribution, and direction), is necessary to determine the role of CFM in the future of tES. Though progress is limited without the inclusion of modelled functional state, CFM-informed studies will bring us closer to knowing whether CFMs improve tES application and by extension, tES efficacy.

As CFM improves and develops, it is likely that it will also become more complex. With this progress comes a risk that features which originally popularised tES, namely that it is inexpensive and easy to use, will be lost. For example, individualised CFM alone requires a high-resolution structural MRI of a subject's brain, specialist software, and user expertise. The increased control of E-field achieved in simulations will require complex multi-electrode tES and neuronavigation equipment for implementation in practical settings, which is not yet readily available. The evolving demands of informed CFM user decision-making, the need for quality MRI scans, accounting for patient-specific changes in neuroanatomy and underlying brain state, and a link between CFM and physiological models of stimulation outcomes, may render the widespread use of tES in clinical settings impractical.

If CFM in its current form is found to improve tES outcomes by allowing users to predict current flow and achieve targeted, individualised stimulation, the benefits of its use must outweigh the burden of additional equipment, expertise, and time required to implement it. Without these gains, the future of tES as an effective, practical, and financially viable therapeutic technique is uncertain.

Funding This work was supported by Brain Research UK (Grant number: 201617-03) and Dunhill Medical Trust [RPGF1810193].

Availability of Data and Material Not applicable.

Code Availability Not applicable.

\section{Compliance with Ethical Standards}

Conflict of Interest The authors declare no competing interests.

Open Access This article is licensed under a Creative Commons Attribution 4.0 International License, which permits use, sharing, adaptation, distribution and reproduction in any medium or format, as long as you give appropriate credit to the original author(s) and the source, provide a link to the Creative Commons licence, and indicate if changes were made. The images or other third party material in this article are included in the article's Creative Commons licence, unless indicated otherwise in a credit line to the material. If material is not included in the article's Creative Commons licence and your intended use is not permitted by statutory regulation or exceeds the permitted use, you will need to obtain permission directly from the copyright holder. To view a copy of this licence, visit http://creativecommons.org/licenses/by/4.0/.

\section{References}

1. Antal A, Alekseichuk I, Bikson M, Brockmöller J, Brunoni AR, Chen $\mathrm{R}$, et al. Low intensity transcranial electric stimulation: safety, ethical, legal regulatory and application guidelines. Clin Neurophysiol. 2017;128:1774-809.

2. Nitsche MA, Paulus W. Excitability changes induced in the human motor cortex by weak transcranial direct current stimulation. J Physiol. 2000;527:633-9.

3. Woods AJ, Antal A, Bikson M, Boggio PS, Brunoni AR, Celnik $\mathrm{P}$, et al. A technical guide to tDCS, and related non-invasive brain stimulation tools. Clin Neurophysiol. 2016;127:1031-48.

4. Goodwill AM, Lum JAG, Hendy AM, Muthalib M, Johnson L, Albein-Urios N, et al. Using non-invasive transcranial stimulation to improve motor and cognitive function in Parkinson's disease: a systematic review and meta-analysis. Sci Rep [Internet]. 2017 [cited 2021 Jun 16];7. Available from: https://www.ncbi. nlm.nih.gov/pmc/articles/PMC5665996/

5. Di Pino G, Pellegrino G, Assenza G, Capone F, Ferreri F, Formica D, et al. Modulation of brain plasticity in stroke: a novel model for neurorehabilitation. Nat Rev Neurol Nature Publishing Group. 2014;10:597-608.

6. Elsner B, Kugler J, Pohl M, Mehrholz J. Transcranial direct current stimulation (tDCS) for improving activities of daily living, and physical and cognitive functioning, in people after stroke. Cochrane Database Syst Rev. 2020;11:CD009645.

7. Ward NS. Non-invasive brain stimulation for stroke recovery: ready for the big time? J Neurol Neurosurg Psychiatry. BMJ Publishing Group Ltd. 2016;87:343-4.

8. Brunoni AR, Amadera J, Berbel B, Volz MS, Rizzerio BG, Fregni F. A systematic review on reporting and assessment of adverse effects associated with transcranial direct current stimulation. Int J Neuropsychopharmacol. 2011;14:1133-45.

9. Brunoni AR, Boggio PS, De Raedt R, Benseñor IM, Lotufo PA, Namur V, et al. Cognitive control therapy and transcranial direct current stimulation for depression: a randomized, doubleblinded, controlled trial. J Affect Disord. 2014;162:43-9.

10. Loo CK, Husain MM, McDonald WM, Aaronson S, O'Reardon JP, Alonzo A, et al. International randomized-controlled trial of transcranial direct current stimulation in depression. Brain stimulation: basic, translational, and clinical research in neuromodulation. Elsevier; 2018;11:125-33.

11. Ekhtiari H, Tavakoli H, Addolorato G, Baeken C, Bonci A, Campanella $\mathrm{S}$, et al. Transcranial electrical and magnetic stimulation (tES and TMS) for addiction medicine: a consensus paper on the present state of the science and the road ahead. Neurosci Biobehav Rev. 2019;104:118-40.

12. Yavari F, Shahbabaie A, Leite J, Carvalho S, Ekhtiari H, Fregni F. Chapter 17 - Noninvasive brain stimulation for addiction medicine: from monitoring to modulation. In: Ekhtiari $\mathrm{H}$, Paulus MP, editors. Progress in Brain Research [Internet]. Elsevier; 2016 [cited 2021 Jun 11]. p. 371-99. Available from: https:// www.sciencedirect.com/science/article/pii/S00796123150014 54

13. Buss SS, Fried PJ, Pascual-Leone A. Therapeutic noninvasive brain stimulation in alzheimer's disease and related dementias. Curr Opin Neurol. 2019;32:292-304.

14. Chew T, Ho K-A, Loo CK. Inter- and Intra-individual variability in response to transcranial direct current stimulation (tDCS) at varying current intensities. Brain Stimul. 2015;8:1130-7.

15. Fekete T, Nikolaev AR, De Knijf F, Zharikova A, van Leeuwen C. Multi-Electrode Alpha tACS During Varying Background Tasks Fails to Modulate Subsequent Alpha Power. Front Neurosci [Internet]. 2018 [cited 2021 Jun 16];12. Available from: https://www. ncbi.nlm.nih.gov/pmc/articles/PMC6026647/ 
16. Wiethoff S, Hamada M, Rothwell JC. Variability in response to transcranial direct current stimulation of the motor cortex. Brain Stimul. 2014;7:468-75.

17. Wörsching J, Padberg F, Ertl-Wagner B, Kumpf U, Kirsch B, Keeser D. Imaging transcranial direct current stimulation (tDCS) of the prefrontal cortex - correlation or causality in stimulationmediated effects? Neurosci Biobehav Rev. 2016;69:333-56.

18. Datta A, Bansal V, Diaz J, Patel J, Reato D, Bikson M. Gyriprecise head model of transcranial direct current stimulation: improved spatial focality using a ring electrode versus conventional rectangular pad. Brain stimulation: basic, translational, and clinical research in neuromodulation. 2009;2:201-207.e1.

19. Faria P, Hallett M, Miranda PC. A finite element analysis of the effect of electrode area and inter-electrode distance on the spatial distribution of the current density in tDCS. Journal of Neural Engineering. 2011;8:066017.

20. Rush S, Driscoll DA. Current Distribution in the brain from surface electrodes: Anesthesia \& Analgesia. 1968;47:717???723.

21. Huang Y, Dmochowski JP, Su Y, Datta A, Rorden C, Parra LC. Automated MRI segmentation for individualized modeling of current flow in the human head. J Neural Eng. 2013;10:066004.

22. Wagner TA, Zahn M, Grodzinsky AJ, Pascual-Leone A. Threedimensional head model Simulation of transcranial magnetic stimulation. IEEE Trans Biomed Eng. 2004;51:1586-98.

23. Miranda PC, Correia L, Salvador R, Basser PJ. Tissue heterogeneity as a mechanism for localized neural stimulation by applied electric fields. Phys Med Biol. 2007;52:5603-17.

24. Datta A, Elwassif M, Battaglia F, Bikson M. Transcranial current stimulation focality using disc and ring electrode configurations: FEM analysis. J Neural Eng. 2008;5:163-74.

25. Miranda PC, Lomarev M, Hallett M. Modeling the current distribution during transcranial direct current stimulation. Clin Neurophysiol. 2006;117:1623-9.

26. Dmochowski JP, Bikson M, Parra LC. The point spread function of the human head and its implications for transcranial current stimulation. Phys Med Biol. 2012;57:6459-77.

27. Dannhauer M, Brooks D, Tucker D, MacLeod R. A pipeline for the simulation of transcranial direct current stimulation for realistic human head models using SCIRun/BioMesh3D. Conf Proc IEEE Eng Med Biol Soc [Internet]. 2012 [cited 2018 May 30];2012. Available from: https://www.ncbi.nlm.nih.gov/pmc/ articles/PMC3651514/

28. Dmochowski JP, Datta A, Bikson M, Su Y, Parra LC. Optimized multi-electrode stimulation increases focality and intensity at target. Journal of Neural Engineering. 2011;8:046011.

29. Dmochowski JP, Datta A, Huang Y, Richardson JD, Bikson M, Fridriksson J, et al. Targeted transcranial direct current stimulation for rehabilitation after stroke. Neuroimage. 2013;75:12-9.

30. Huang Y, Datta A, Bikson M, Parra LC. ROAST: an opensource, fully-automated, realistic volumetric-approach-based simulator For TES. 2018 40th Annual International Conference of the IEEE Engineering in Medicine and Biology Society (EMBC) [Internet]. Honolulu, HI: IEEE; 2018 [cited 2021 Feb 12]. p. 3072-5. Available from: https://ieeexplore.ieee.org/ document/8513086/

31. Huang Y, Datta A, Bikson M, Parra LC. Realistic volumetric-approach to simulate transcranial electric stimulationROAST - a fully automated open-source pipeline. J Neural Eng. 2019;16:056006.

32. Huang Y, Thomas C, Datta A, Parra LC. Optimized tDCS for targeting multiple brain regions: an integrated implementation. 2018 40th Annual International Conference of the IEEE Engineering in Medicine and Biology Society (EMBC). 2018. p. 3545-8.
33. Lee C, Jung Y-J, Lee SJ, Im C-H. COMETS2: an advanced MAT$\mathrm{LAB}$ toolbox for the numerical analysis of electric fields generated by transcranial direct current stimulation. J Neurosci Methods. 2017:277:56-62.

34. Saturnino GB, Puonti O, Nielsen JD, Antonenko D, Madsen KH, Thielscher A. SimNIBS 2.1: a comprehensive pipeline for individualized electric field modelling for transcranial brain stimulation. In: Makarov S, Horner M, Noetscher G, editors. Brain and human body modeling: computational human modeling at EMBC 2018 [Internet]. Cham: Springer International Publishing; 2019 [cited 2021 Jun 16]. p. 3-25. Available from: https://doi.org/10. 1007/978-3-030-21293-3_1.

35. Huang Y, Liu AA, Lafon B, Friedman D, Dayan M, Wang X, et al. Measurements and models of electric fields in the in vivo human brain during transcranial electric stimulation. 2017;26.

36. Huang Y, Liu AA, Lafon B, Friedman D, Dayan M, Wang X, et al. Correction: Measurements and models of electric fields in the in vivo human brain during transcranial electric stimulation. eLife Sciences. 2018;7:e35178.

37. Koessler L, Colnat-Coulbois S, Cecchin T, Hofmanis J, Dmochowski JP, Norcia AM, et al. In-vivo measurements of human brain tissue conductivity using focal electrical current injection through intracerebral multicontact electrodes. Hum Brain Mapp. 2016;38:974-86.

38. Opitz A, Falchier A, Yan C-G, Yeagle EM, Linn GS, Megevand $\mathrm{P}$, et al. Spatiotemporal structure of intracranial electric fields induced by transcranial electric stimulation in humans and nonhuman primates. Scientific Reports [Internet]. 2016 [cited 2018 Jun 12];6. Available from: http://www.nature.com/articles/srep3 1236

39. Laakso I, Tanaka S, Koyama S, Santis VD, Hirata A. Inter-subject variability in electric fields of motor cortical tDCS. Brain stimulation: basic, translational, and clinical research in neuromodulation. Elsevier; 2015;8:906-13.

40. Polanía R, Nitsche MA, Ruff CC. Studying and modifying brain function with non-invasive brain stimulation. Nat Neurosci Nature Publishing Group. 2018;21:174-87.

41. Evans C, Bachmann C, Lee JSA, Gregoriou E, Ward N, Bestmann $\mathrm{S}$. Dose-controlled tDCS reduces electric field intensity variability at a cortical target site. Brain Stimul. 2020;13:125-36.

42. Laakso I, Mikkonen M, Koyama S, Hirata A, Tanaka S. Can electric fields explain inter-individual variability in transcranial direct current stimulation of the motor cortex? Sci Rep. Nature Publishing Group; 2019;9:626.

43. Datta A, Truong D, Minhas P, Parra LC, Bikson M, Inter-individual variation during transcranial direct current stimulation and normalization of dose using MRI-Derived computational models. Frontiers in Psychiatry [Internet]. [cited 2018 Jun 12];3. Available from. 2012. https://doi.org/10.3389/fpsyt.2012.00091/abstract.

44. Esmaeilpour Z, Marangolo P, Hampstead BM, Bestmann S, Galletta E, Knotkova H, et al. Incomplete evidence that increasing current intensity of tDCS boosts outcomes. Brain Stimul. 2018;11:310-21.

45. Lisman JE. Three Ca2+ levels affect plasticity differently: the LTP zone, the LTD zone and no man's land. J Physiol. 2001;532:285.

46. Bikson M, Inoue M, Akiyama H, Deans JK, Fox JE, Miyakawa H, et al. Effects of uniform extracellular DC electric fields on excitability in rat hippocampal slices in vitro: modulation of neuronal function by electric fields. J Physiol. 2004;557:175-90.

47. Bikson M, Rahman A, Datta A. Computational models of transcranial direct current stimulation. Clin EEG Neurosci. 2012;43:176-83. 
48. Edwards D, Cortes M, Datta A, Minhas P, Wassermann EM, Bikson M. Physiological and modeling evidence for focal transcranial electrical brain stimulation in humans: a basis for high-definition tDCS. Neuroimage. 2013;74:266-75.

49. Saturnino GB, Antunes A, Thielscher A. On the importance of electrode parameters for shaping electric field patterns generated by tDCS. Neuroimage. 2015;120:25-35.

50. Mikkonen M, Laakso I, Tanaka S, Hirata A. Cost of focality in TDCS: interindividual variability in electric fields. Brain Stimul. 2020;13:117-24.

51. Farahani F, Kronberg G, FallahRad M, Oviedo HV, Parra LC. Effects of direct current stimulation on synaptic plasticity in a single neuron. Brain stimulation: basic, translational, and clinical research in neuromodulation [Internet]. Elsevier; 2021 [cited 2021 Mar 23];0. Available from: https://www.brainstimjrnl.com/article/ S1935-861X(21)00048-6/abstract

52. Lafon B, Rahman A, Bikson M, Parra LC. Direct current stimulation alters neuronal input/output function. Brain stimulation: basic, translational, and clinical research in neuromodulation. Elsevier; 2017;10:36-45.

53. Rahman A, Reato D, Arlotti M, Gasca F, Datta A, Parra LC, et al. Cellular effects of acute direct current stimulation: somatic and synaptic terminal effects. J Physiol. 2013;591:2563-78.

54. Hannah R, Iacovou A, Rothwell JC. Direction of TDCS current flow in human sensorimotor cortex influences behavioural learning. Brain stimulation: basic, translational, and clinical research in neuromodulation. Elsevier; 2019;12:684-92.

55. Rawji V, Ciocca M, Zacharia A, Soares D, Truong D, Bikson M, et al. tDCS changes in motor excitability are specific to orientation of current flow. Brain Stimul. 2018;11:289-98.

56. Antonenko D, Grittner U, Saturnino G, Nierhaus T, Thielscher A, Flöel A. Inter-individual and age-dependent variability in simulated electric fields induced by conventional transcranial electrical stimulation. NeuroImage. 2021;224:117413.

57. Ciechanski P, Carlson HL, Yu SS, Kirton A. Modeling transcranial direct-current stimulation-induced electric fields in children and adults. Front Hum Neurosci [Internet]. Frontiers; 2018 [cited 2021 Jun 17];12. Available from: https://www.frontiersin.org/ articles/https://doi.org/10.3389/fnhum.2018.00268/full

58. Indahlastari A, Albizu A, O'Shea A, Forbes MA, Nissim NR, Kraft JN, et al. Modeling transcranial electrical stimulation in the aging brain. Brain Stimul. 2020;13:664-74.

59. Minhas P, Bikson M, Woods A, Rosen A, Kessler S. Transcranial direct current stimulation in pediatric brain: a computational modeling study. Conference proceedings : Annual International Conference of the IEEE Engineering in Medicine and Biology Society IEEE Engineering in Medicine and Biology Society Conference. 2012;2012:859-62.

60. Johnstone A, Zich C, Evans C, Lee J, Ward N, Bestmann $\mathrm{S}$. The impact of brain lesions on tDCS-induced electric field magnitude. bioRxiv. Cold Spring Harbor Laboratory; 2021;2021.03.19.436124.

61. Minjoli S, Saturnino GB, Blicher JU, Stagg CJ, Siebner HR, Antunes A, et al. The impact of large structural brain changes in chronic stroke patients on the electric field caused by transcranial brain stimulation. NeuroImage: Clinical. 2017;15:106-17.

62. Mosayebi-Samani M, Jamil A, Salvador R, Ruffini G, Haueisen J, Nitsche MA. The impact of individual electrical fields and anatomical factors on the neurophysiological outcomes of tDCS: a TMS-MEP and MRI study. Brain Stimul. 2021;14:316-26.

63. Opitz A, Paulus W, Will S, Antunes A, Thielscher A. Determinants of the electric field during transcranial direct current stimulation. Neuroimage. 2015;109:140-50.
64. Rosso C, Valabregue R, Arbizu C, Ferrieux S, Vargas P, Humbert $\mathrm{F}$, et al. Connectivity between right inferior frontal gyrus and supplementary motor area predicts after-effects of right frontal cathodal tDCS on picture naming speed. Brain stimulation: basic, translational, and clinical research in neuromodulation. Elsevier; 2014;7:122-9.

65. Laakso I, Mikkonen M, Koyama S, Ito D, Yamaguchi T, Hirata A, Electric field dependent effects of motor cortical TDCS., et al. cited 2018 Jun 5. Available from. 2018. https://doi.org/10.1101/ 327361.

66. Antal A, Terney D, Poreisz C, Paulus W. Towards unravelling task-related modulations of neuroplastic changes induced in the human motor cortex. Eur J Neurosci. 2007;26:2687-91.

67. Horvath JC, Carter O, Forte JD, Transcranial direct current stimulation: five important issues we aren't discussing (but probably should be). Front Syst Neurosci [Internet]. Frontiers,. [cited 2021 Jun 18];8. Available from. 2014. https://doi.org/10.3389/fnsys. 2014.00002/full.

68. Miyaguchi S, Onishi H, Kojima S, Sugawara K, Tsubaki A, Kirimoto $\mathrm{H}$, et al. Corticomotor excitability induced by anodal transcranial direct current stimulation with and without non-exhaustive movement. Brain Res. 2013;1529:83-91.

69. Paulus W, Antal A, Nitsche MA. Chapter 4: Physiological basis and methodological aspects of transcranial electric stimulation (tDCS, tACS, and tRNS). Transcranial Brain Stimulation. 2012;20.

70. Bachtiar V, Near J, Johansen-Berg H, Stagg CJ. Modulation of GABA and resting state functional connectivity by transcranial direct current stimulation. Elife. 2015;4:e08789.

71. Bachtiar V, Johnstone A, Berrington A, Lemke C, Johansen-Berg $\mathrm{H}$, Emir U, et al. Modulating regional motor cortical excitability with noninvasive brain stimulation results in neurochemical changes in bilateral motor Cortices. J Neurosci. 2018;38:7327-36.

72. Amadi U, Allman C, Johansen-Berg H, Stagg CJ. The homeostatic interaction between anodal transcranial direct current stimulation and motor learning in humans is related to GABAA activity. Brain Stimul. 2015;8:898-905.

73. Kim S, Stephenson MC, Morris PG, Jackson SR. tDCS-induced alterations in GABA concentration within primary motor cortex predict motor learning and motor memory: a $7 \mathrm{~T}$ magnetic resonance spectroscopy study. Neuroimage. 2014;99:237-43.

74. Stagg CJ, Best JG, Stephenson MC, O’Shea J, Wylezinska M, Kincses ZT, et al. Polarity-sensitive modulation of cortical neurotransmitters by transcranial stimulation. J Neurosci. 2009;29:5202-6.

75. Pellegrini M, Zoghi M, Jaberzadeh S. Can genetic polymorphisms predict response variability to anodal transcranial direct current stimulation of the primary motor cortex? Eur J Neurosci. 2021;53:1569-91.

76. Stagg CJ, Antal A, Nitsche MA. Physiology of transcranial direct current stimulation: The Journal of ECT. 2018;34:144-52.

77. Filmer HL, Ehrhardt SE, Bollmann S, Mattingley JB, Dux PE. Accounting for individual differences in the response to tDCS with baseline levels of neurochemical excitability. Cortex. 2019;115:324-34.

78. Aberra AS, Wang B, Grill WM, Peterchev AV. Simulation of transcranial magnetic stimulation in head model with morphologically-realistic cortical neurons. Brain stimulation: basic, translational, and clinical research in neuromodulation. Elsevier; 2020;13:175-89.

79. Bonaiuto JJ, Berker A de, Bestmann S. Response repetition biases in human perceptual decisions are explained by activity decay in 
competitive attractor models. Ivry R, editor. eLife. eLife Sciences Publications, Ltd; 2016;5:e20047.

80. Bonaiuto JJ, Bestmann S. Chapter 4 - Understanding the nonlinear physiological and behavioral effects of tDCS through computational neurostimulation. In: Bestmann S, editor. Progress in Brain Research [Internet]. Elsevier; 2015 [cited 2021 Jun 22]. p. 75-103.
Available from: https://www.sciencedirect.com/science/article/pii/ S0079612315001041

81. McCann H, Pisano G, Beltrachini L. Variation in Reported Human Head Tissue Electrical Conductivity Values. Brain Topogr. 2019;32:825-58.

Publisher's Note Springer Nature remains neutral with regard to jurisdictional claims in published maps and institutional affiliations. 